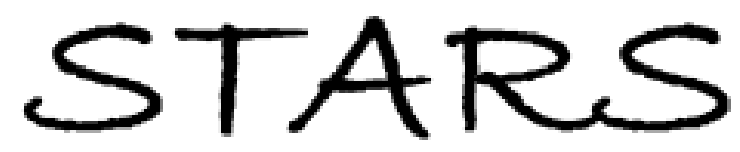

University of Central Florida

STARS

$1-1-2008$

\title{
Hartree-Fock studies of the triple differential cross section for electron-impact ionization of the hydrogen atom
}

Hari P. Saha

University of Central Florida

Find similar works at: https://stars.library.ucf.edu/facultybib2000 University of Central Florida Libraries http://library.ucf.edu

This Article is brought to you for free and open access by the Faculty Bibliography at STARS. It has been accepted for inclusion in Faculty Bibliography 2000 s by an authorized administrator of STARS. For more information, please contact STARS@ucf.edu.

\section{Recommended Citation}

Saha, Hari P., "Hartree-Fock studies of the triple differential cross section for electron-impact ionization of the hydrogen atom" (2008). Faculty Bibliography 2000s. 917.

https://stars.library.ucf.edu/facultybib2000/917

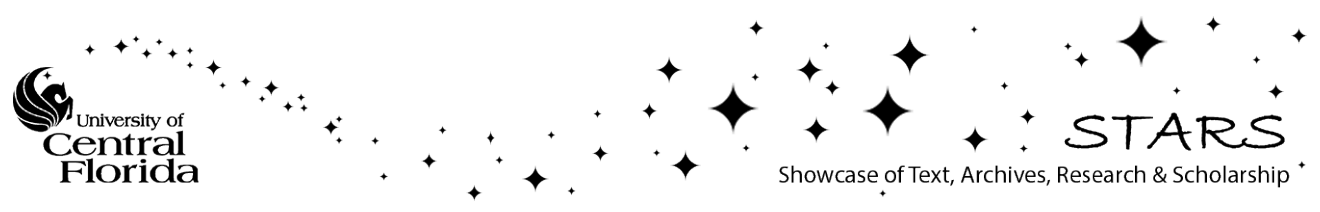




\title{
Hartree-Fock studies of the triple differential cross section for electron-impact ionization of the hydrogen atom
}

\author{
Hari P. Saha* \\ Physics Department, University of Central Florida, Orlando, Florida 32816, USA
}

(Received 20 February 2008; published 13 June 2008)

\begin{abstract}
We report absolute triple differential cross sections in coplanar $\theta_{12}=\pi$ geometry for the ionization of atomic hydrogen by electron impact at low energies. We used our recently extended multiconfiguration Hartree-Fock method successfully to calculate triple differential cross sections of $\mathrm{H}$ for incident energies $E_{0}$ of 14.6, 15.6, 17.6, 20, and $25 \mathrm{eV}$ for equal energy sharing of excess energies by the two final state continuum electrons. It has been shown that there is very little difference between the results obtained in the Hartree-Fock and the screening potential approximations implying that in the case of $\mathrm{H}$ and $\theta_{12}=\pi$ configuration distortion effects between the two outgoing electrons are small even at low energies. Our results are compared with available absolute and relative experimental data and other existing theoretical calculations and are found to be in very good agreement.
\end{abstract}

DOI: 10.1103/PhysRevA.77.062705

PACS number(s): 34.80.Dp

\section{INTRODUCTION}

The electron-impact ionization processes are of fundamental importance for understanding the dynamics of threebody systems interacting through Coulomb forces. Nowadays there are a large number of experimental data available for low energy triple differential cross sections for various targets. Accurate analysis of these data would be a direct test of any theory. There have been a few theoretical calculations available on triple differential cross sections for $\mathrm{H}$ target at low electron-impact energies. The main emphasis has been on evaluating the final total wave functions accurately. Among several theoretical approaches, which produce reliable results, are the convergent close coupling (CCC) method [1], the exterior complex scaling (ECS) approach [2], the time-dependent close coupling (TDCC) method [3], the multichannel $R$-matrix theory [4], the distorted wave method [5], and the distorted partial wave approach [6]. In addition, Brauner et al. [7,8] applied the exact asymptotic wave functions and reported results of triply differential cross sections for electron-impact ionization of $\mathrm{H}$ and $\mathrm{He}$ atoms at low and intermediate energies. Temkin [9] and Poet [10] also investigated the electron-impact ionization of atoms. Their approach is normally used to test the results obtained by other theories. Temkin [11] also developed a dipole theory to investigate the threshold behavior of electronimpact ionization of atoms. On the experimental side there are also several experimental data available at low excess energies. The relative triple differential cross section for $\mathrm{H}$ target are measured by Schlemmer et al. [12] for excess energy of $4 \mathrm{eV}$ in which the two continuum electrons leave in opposite directions. Roder et al. $[13,14]$ measured the absolute triple differential cross sections for $\mathrm{H}$ for incident energies of 15.6 and $17.6 \mathrm{eV}$ and relative triple differential cross sections at incident electron energy 14.6, 20, and $25 \mathrm{eV}$ for equal energy sharing by the two final-state continuum electrons and $\theta_{12}=\pi$ configuration where $\theta_{12}$ is the angle be-

\footnotetext{
*hps1@physics.ucf.edu
}

tween the two outgoing continuum electrons.

In an earlier paper [15] we reported results of a triple differential cross section of $\mathrm{H}$ only for the case of $4 \mathrm{eV}$ excess energy shared both equally and unequally by the two final state continuum electrons for $\theta_{12}=\pi$ geometry and compared with relative experimental results of Schlemmer et al. [12]. The purpose of this investigation is to find how Hartree-Fock (HF) approximation compares with other most accurate methods when the two final state continuum electrons leave in the opposite direction. In this paper we present results of our calculation on triple differential cross sections at several incident electron energies using the $\mathrm{HF}$ and the screening potential (SP) approximations [16-18] to compare with available absolute and relative experimental observations and other accurate theoretical calculations. In all of the calculations presented, the two final state electrons share the excess energy equally and scattered in the opposite direction. Our results are compared with experimental data of Roder et al. $[13,14]$ and theoretical results of Pan and Starace [6] calculated using distorted partial wave approximation as well as those obtained by Bray $[1,19,20]$ who used convergent close coupling approach, by Baertschy et al. [21] calculated using the exterior complex scaling approach and by Colgan and Pindzola [22] who used the time-dependent close coupling approximation.

\section{THEORY}

The theory is described in our previous paper [15]. Briefly, the triple differential cross section for electronimpact ionization of atoms is given by [6]

$$
\frac{d^{3} \sigma}{d E_{2} d \Omega_{1} d \Omega_{2}}=\frac{(2 \pi)^{4}}{k} k_{1} k_{2}\left|\left\langle\Psi_{f}^{-}|V| \Phi_{i}^{+}\right\rangle\right|^{2},
$$

where $\Phi_{i}^{+}$and $\Psi_{f}^{-}$are, respectively, the initial and the final state wave functions of the system, $\vec{k}$ is the momentum of the incident electron, and $\vec{k}_{1}$ and $\vec{k}_{2}$ are the momenta of the two continuum electrons in the final state. $d \Omega_{1}$ and $d \Omega_{2}$ are the solid angles associated with the two continuum electrons in 
the final state. $E_{i}=\frac{k_{i}^{2}}{2}$ is the kinetic energy of the $i$ th final state continuum electron. The perturbation $V$ is defined as

$$
V=\sum_{i=1}^{N} \frac{1}{\left|r_{N+1}-r_{i}\right|}-V_{H F}^{N+1}\left(r_{N+1}\right) .
$$

The first term describes the Coulomb interaction between the incident electron and the $N$ target electrons and the second term is a multiconfiguration Hartree-Fock approximation to this interaction which is used to construct and describe approximately the initial state $\Phi_{i}^{+}$. The initial state $\Phi_{i}^{+}$is expanded in terms of the antisymmetrized $L S$ coupled wave function of the $N$ electron target characterized by the orbital and spin angular momentum $L_{0}$ and $S_{0}$ and the single electron wave function of the incoming electron having momentum $\vec{k}$ whereas the final state $\Psi_{f}^{-}$is characterized by the orbital and spin angular momenta $L_{c}$ and $S_{c}$ of the $(N-1)$ electron and by the momenta $\vec{k}_{1}$ and $\vec{k}_{2}$ and orbital angular momenta $l_{1}, l_{2}$ of the two continuum electrons to form the total $L S$ state.

As mentioned earlier we have carried out calculations of the final continuum electron wave functions in the two approximations. In the screening potential approximation the exact Coulomb interaction between the two continuum electrons in the final state is replaced by a variationally determined screening potential [16-18] whereas in the HF approximation we ignored this interaction between the two final state continuum electrons. The difference between the wave functions calculated with these two approximations will determine the effect of distortion of the final state wave functions. For the configuration considered here, $\widehat{k_{1}}=-\widehat{k_{2}}$, the screening potential for the two continuum electrons are determined by the screening charges [18]

$$
\Delta_{i}=\frac{k_{i}^{2}}{\left(k_{1}+k_{2}\right)^{2}} \quad(i=1,2) \text {. }
$$

\section{A. Wave functions for the continuum electrons}

The multichannel multiconfiguration Hartree-Fock (MCHF) method was described earlier [23]. The total wave function in the HF approximation [24] at energy $E=E_{i}$ $+k^{2} / 2$ and term value $L S$ can be expressed as [23]

$$
\Psi_{E}=\Phi\left(\gamma_{i} L_{i} S_{i} ; N\right) F_{k_{i} l_{i}},
$$

where $\Phi\left(\gamma_{i} L_{i} S_{i} ; N\right)$ represents $N$ electron target wave function having energy $E_{i}$, configuration $\gamma_{i}$, and the term $L_{i}$ and $S_{i}$ coupled with a single electron wave function $F_{k_{i} l_{i}}$ having energy $\frac{1}{2} k_{i}^{2}$ (in atomic units) and orbital angular momentum $l_{i}$ to form an antisymmetric configuration for the $(N+1)$ electron system with a designated term value. The above wave function is defined in terms of a set of radial functions $P_{i}(r), i=1, \ldots, m$, as, for example, $F_{k_{i} l_{i}}=\frac{P_{i}(r)}{r} Y_{l_{i} m_{i}}\left(\theta_{i}, \phi_{i}\right) \chi_{m_{s}}$ where $Y_{l_{i} m_{i}}\left(\theta_{i}, \phi_{i}\right)$ is the spherical harmonic and $\chi_{m_{s}}$ is the spin function. The set of radial functions $P_{i}(r), i=1, \ldots, m_{t}$ describing the targets are obtained from the HF bound state calculations for the targets and are kept fixed. The set of radial functions describing the continuum orbitals are determined variationally. These radial functions are the solutions of the integrodifferential equations of the form [23]

$$
\begin{aligned}
{\left[\frac{d^{2}}{d r^{2}}+\frac{2 Z}{r}-\frac{l_{i}\left(l_{i}+1\right)}{r^{2}}\right] P_{i}(r)=} & \frac{2}{r}\left[Y_{i}(r) P_{i}(r)+X_{i}(r)+I_{i}(r)\right] \\
& +\sum_{i^{\prime}} \epsilon_{i i^{\prime}} P_{i^{\prime}}(r)
\end{aligned}
$$

which has the same form as the Hartree-Fock equation for a singly occupied orbital of a bound state system, the only difference being the specified binding energy $\epsilon_{i i}=\frac{k^{2}}{2}$ and the boundary condition at infinity.

In this equation $\frac{2}{r} Y_{i}(r)$ is a part of the direct potential, $\frac{2}{r} X_{i}(r)$ is the exchange function, and $\frac{2}{r} I_{i}(r)$ represents terms arising from interactions between the configurations. The off-diagonal energy parameters $\epsilon_{i i}$ are related to Lagrange multipliers that ensure orthogonality between the continuum and the bound electrons of the target having the same symmetry. These operators have their usual meanings [25] as for bound state problems.

In the single channel case, the radial function $P_{i}(r)$ satisfies the boundary conditions

$$
P_{i}(r) \underset{r \rightarrow 0}{\rightarrow} r^{l+1}, \quad P_{i}(r) \underset{r \rightarrow+\infty}{\rightarrow} \sqrt{\frac{2}{\pi k_{i}}} \sin \left(k_{i} r-\frac{l_{i} \pi}{2}+\delta_{l}\right)
$$

if the target is an atom and

$$
P_{i}(r) \underset{r \rightarrow+\infty}{\rightarrow} \sqrt{\frac{2}{\pi k_{i}}} \sin \left(k_{i} r-\frac{l_{i} \pi}{2}+\frac{q}{k_{i}} \ln 2 k_{i} r+\sigma_{l}+\delta_{l}\right)
$$

if the target is an ion. Here $\sigma_{l}=\arg \left[\Gamma\left(l_{i}+1-\frac{i q}{k_{i}}\right)\right]$ is the Coulomb phase shift. $q=Z-N$ is the net charge of the ion.

The integrodifferential Eq. (5) is solved numerically by the iterative method similar to the bound state problem [25]. The self-consistent field procedure is applied to compute the continuum wave functions. The continuum radial function is normalized by fitting the computational values at two adjacent points to the regular and irregular Bessel or Coulomb functions depending on the target as soon as the region is reached where the direct and exchange potentials are vanishingly small. This may be at a considerably smaller value of $r$ than the asymptotic form represented by the boundary conditions specified in Eqs. (6) and (7).

\section{B. Approximations used to calculate initial and the final state wave functions}

In this paper, we have considered the HF and the screening potential approximations to determine the effect of distortion between the two final state continuum electrons.

Initial state. As already mentioned, the initial state wave function $\Phi_{i}^{+}$is expanded in terms of the antisymmetrized $L S$ coupled wave function of the $N$ electron target and the single electron wave function of the incident electron. In the present case, the initial target state is the hydrogen atom whose wave function is known analytically. The continuum radial wave functions are calculated by solving the integrodifferential Eq. (5) with the HF potential of the target hydro- 
gen atom under the specified boundary conditions, where the target electron wave function is kept frozen. In the zero iteration we put $X_{i}(r)=0$ and solve Eq. (5) numerically for the wave function $P_{i}(r)$. Then use this wave function to construct $X_{i}(r)$ and solve the integrodifferential equation iteratively self-consistently until it is converged.

Final state. The wave function for the final state continuum electrons are calculated in both HF and the screening potential approximations. In the screening potential approximation each of the continuum electron wave functions is calculated in the variationally determined screening potential due to the mutual screening interactions [16]-[18]. For the configurations considered here, $\widehat{k_{1}}=-\widehat{k_{2}}$, the screening potentials for the two continuum electrons are obtained by the screening charges Eq. (3). In the HF approximation the radial wave function of each of the two final state continuum electrons is obtained by solving Eq. (5) with the potential of the singly charged hydrogen atom whereas in the screening potential approximation Eq. (5) is solved with $X_{i}(r)=I_{i}(r)=0$ and $\frac{Y_{i}(r)}{r}=\Delta_{i} y_{0}(r)$ with the properties that $y_{0} \rightarrow 0$ as $r \rightarrow 0$ and $y_{0} \rightarrow \frac{1}{r}$ as $r \rightarrow \infty$. Specifically, $y_{0}(r)$ is a special case of the general function

$$
\begin{aligned}
y_{\lambda}\left(n l, n^{\prime} l^{\prime}, r\right)= & r^{-(\lambda+1)} \int_{0}^{r} t^{\lambda} P_{n l}(t) P_{n^{\prime} l^{\prime}}(t) d t \\
& +r^{\lambda} \int_{r}^{\infty} t^{-(\lambda+1)} P_{n l}(t) P_{n^{\prime} l^{\prime}}(t) d t .
\end{aligned}
$$

Using the initial and the final state wave functions one obtains the triple differential cross section as [6]

$$
\begin{aligned}
\sigma_{H}^{(3)}= & \frac{\pi}{4 k^{2}} \sum_{L L^{\prime}} \sum_{S} A_{H}(L S) A_{H}^{*}\left(L^{\prime} S\right) \sum_{\lambda}(2 \lambda+1) \\
& \times P_{\lambda}\left(\widehat{k_{1}} \cdot \hat{k}\right)(2 L+1)\left(2 L^{\prime}+1\right)\left(\begin{array}{ccc}
L & L^{\prime} & \lambda \\
0 & 0 & 0
\end{array}\right)^{2},
\end{aligned}
$$

where $L$ and $S$ are the orbital and spin angular momenta of the coupled pair of final-state continuum electrons, $P_{\lambda}$ is a Legendre polynomial with argument $\left(\widehat{k_{1}} \cdot \hat{k}\right)=\cos \theta_{1}, \theta_{1}$ being the angle made by one of the final state continuum electrons with momentum $k_{1}$ with the momentum $\hat{k}$ of the incident electron. The scattering amplitude $A(L S)$ for the $L S$ partial wave is given by [6]

$$
\begin{aligned}
A(L S)= & {\left[\frac{(2 S+1)}{2\left(2 S_{0}+1\right)}\right]^{1 / 2} \sum_{l_{1} l_{2}}(-1)^{l_{1}+L} f\left(l, l_{1} l_{2}\right) } \\
& \times\left[\left(2 l_{1}+1\right)\left(2 l_{2}+1\right)\right]^{1 / 2}\left(\begin{array}{ccc}
l_{1} & l_{2} & L \\
0 & 0 & 0
\end{array}\right)\left\langle\psi_{f}|V| \psi_{i}\right\rangle,
\end{aligned}
$$

where

$$
f\left(l, l_{1}, l_{2}\right)=i^{l+l_{1}+l_{2}} e^{i\left(\delta_{l}+\sigma_{l_{1}}+\delta_{l_{1}}+\sigma_{l_{2}}+\delta_{l_{2}}\right)}
$$

and

$$
\begin{aligned}
\left\langle\psi_{f}|V| \psi_{i}\right\rangle= & (-1)^{l}\left(\left[l_{1}\right]\left[l_{2}\right]\right)^{1 / 2}\left(\begin{array}{ccc}
l_{1} & l_{2} & L \\
0 & 0 & 0
\end{array}\right) \\
& \times\left\{\left[l_{1}\right]^{-1} R^{l_{1}}\left(k_{1} l_{1}, k_{2} l_{2} ; 1 s, k l\right)\right. \\
& \left.+(-1)^{S}\left[l_{2}\right]^{-1} R^{l_{2}}\left(k_{2} l_{2}, k_{1} l_{1} ; 1 s, k l\right)\right\}
\end{aligned}
$$

with $[x]=(2 x+1)$. The Slater integrals are defined by

$$
R^{\lambda}(a, b ; c, d)=\int_{0}^{\infty} P_{b}(r) P_{d}(r) y_{\lambda}(a, c ; r) d r,
$$

where $y_{\lambda}(a, c ; r)$ is defined in Eq. (8).

\section{COMPUTATIONAL PROCEDURE}

In the present case the target considered is the hydrogen atom whose wave function is analytically known. The incident electron wave function in the initial state is calculated in the HF approximation at each of the five kinetic energies $E_{0}=14.6,15.6,17.6,20$, and $25 \mathrm{eV}$ for angular momenta $l$ $=0-6$. The wave functions and the phase shifts are obtained for each angular momentum at each kinetic energy of the incident electron.

To compare with the experimental data $[13,14]$ and the other existing theoretical results [6,19-22] we considered excess energies corresponding to each incident electron energy shared equally between the two final state electrons. Each of the final state continuum electron wave functions is calculated in both the HF and the screening potential approximation. In the HF approximation the wave function of each of the final state continuum electrons is calculated at each kinetic energy and angular momentum from $l=0$ to $l=6$. The wave functions are obtained for each of the kinetic energies $E_{1}=0.5,1.0,2.0,3.2$, and $5.7 \mathrm{eV}$ for angular momentum from $l=0$ to $l=6$. In the screening potential approximation the exact Coulomb interaction between the two continuum electrons is replaced by a variationally determined screening potential due to the mutual screening interaction using effective charges which satisfy proper asymptotic boundary conditions. The radial wave function for each of the two final state continuum electrons for each pair of orbital angular momentum $\left(l_{1}, l_{2}\right)$ and each partial wave $L=0-6$ are calculated using the screening potentials

$$
V_{i}\left(k_{1}, k_{2}\right)=\Delta_{i} Y_{i}(r)
$$

defined in the text. In this case each of the continuum electrons in the final state sees appropriate screening potential. We obtained wave functions and phase shifts of each of the final state continuum electrons for angular momentum $l$ $=0-6$ at each kinetic energy considered.

With the initial state and the final state wave functions we calculated the amplitudes [Eq. (10)] for each partial wave $L S$ in both the HF and the screening potential approximations. Then we calculated the triple differential cross sections at each kinetic energy of the incident electron as a function of scattering angle $\theta_{1}$ of one of the final state electrons in both the $\mathrm{HF}$ and the screening potential approximations using Eq. (9). We tested the convergence of the cross section with respect to the number of angular momentum $\left(l_{1}, l_{2}\right)$ pairs for 


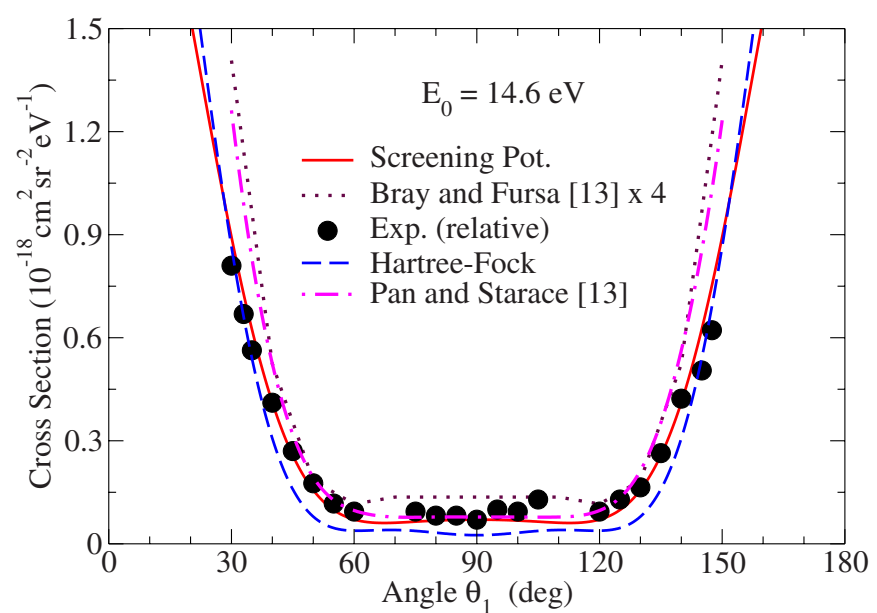

FIG. 1. (Color online) Triple differential cross sections of $\mathrm{H}$ for $\theta_{12}=\pi, E_{0}=14.6 \mathrm{eV}$, and equal energy sharing. Experiment: Roder et al. [13].

$l_{1}=0-6$ and $l_{2}=0-6$ included in each partial wave $L$ as well as convergence with respect to the total number of $L$ partial waves. We found that $L=0-6$ partial waves and a maximum of 15 pairs of $\left(l_{1}, l_{2}\right)$ for each partial wave $L$ are sufficient to obtain converged results of cross section at the low energies of the final state continuum electrons considered here.

\section{RESULTS}

As stated earlier, we consider an equal energy configuration in which the relative angle between the two final state electrons is kept fixed at $\theta_{12}=\pi$ and $\theta_{1}$ is varied. Figures $1-5$ show our calculated results for the equal energy sharing electron-hydrogen triple differential cross section calculated in the $\mathrm{HF}$ and the screening potential approximations for incident energies of $14.6,15.6,17.6,20$, and $25 \mathrm{eV}$. The experimental measurements $[13,14]$ and other available accurate theoretical results for these energies are also presented for comparison. The experimental results at 15.6 and $17.6 \mathrm{eV}$ are absolute. Our theoretical results at these energies calculated in both the approximations compare very well with the

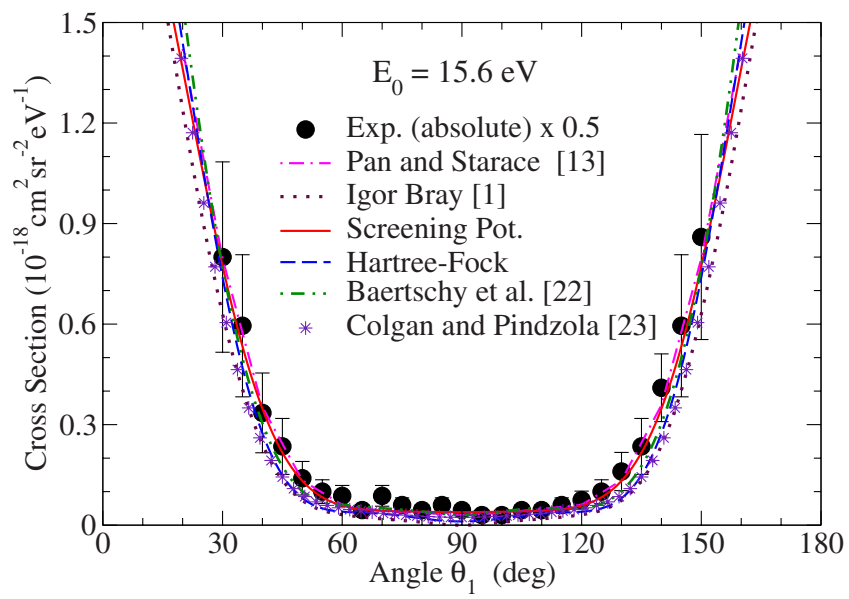

FIG. 2. (Color online) Same as Fig. 1, but for $E_{0}=15.6 \mathrm{eV}$.

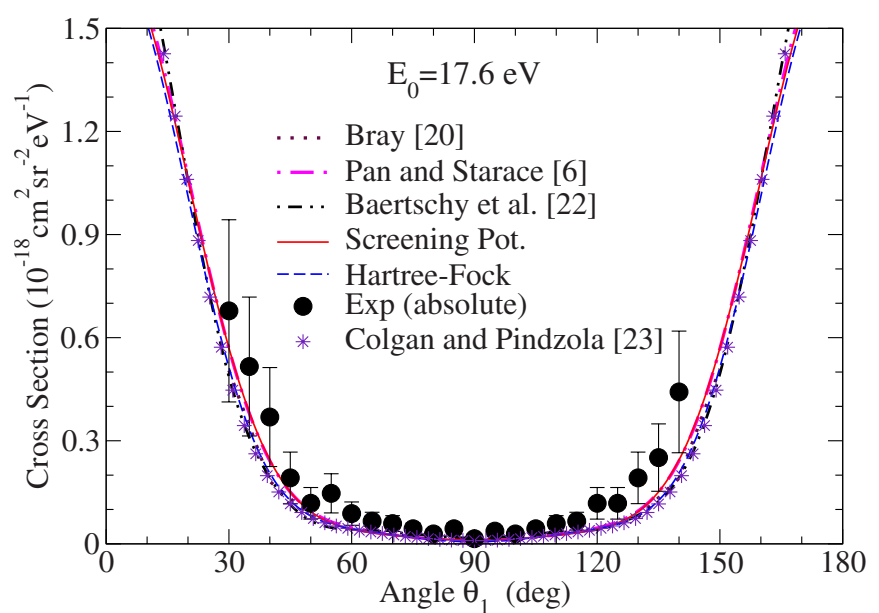

FIG. 3. (Color online) Same as Fig. 1, but for $E_{0}=17.6 \mathrm{eV}$.

absolute experimental data as well as with the convergent close coupling results of Bray $[1,20]$, the exterior complex scaling results of Baertschy et al. [21], and the timedependent close coupling results of Colgan and Pindzola [22]. The error bars on the absolute measurements are $35 \%$ at 15.6 and $40 \%$ at $17.6 \mathrm{eV}$. At $17.6 \mathrm{eV}$ both our $\mathrm{HF}$ and screening potential results are very close and fall within the error bars at all angles indicating that at this energy the effect of distortion between the two final state electrons is very small. Both of our results at $15.6 \mathrm{eV}$ are also very close to each other but the screening potential results are in better agreement with the experiment. The experimental measurement is multiplied by a factor of 0.5 to account for improper experimental normalization as mentioned by Bray [19] and further discussed by Baertschy et al. [21]. We also found that at these energies our screening potential results are in very good agreement with distorted partial wave results of Pan and Starace [13] who used the same approximation and the convergent close coupling results of Bray $[19,20]$, the exterior complex scaling results of Baertschy et al. [21] and the time-dependent close coupling results of Colgan and Pindzola [22]. At all other energies 14.6, 20, and $25 \mathrm{eV}$ the relative experimental measurements are normalized to our screening potential results at the experimental minimum $\theta$

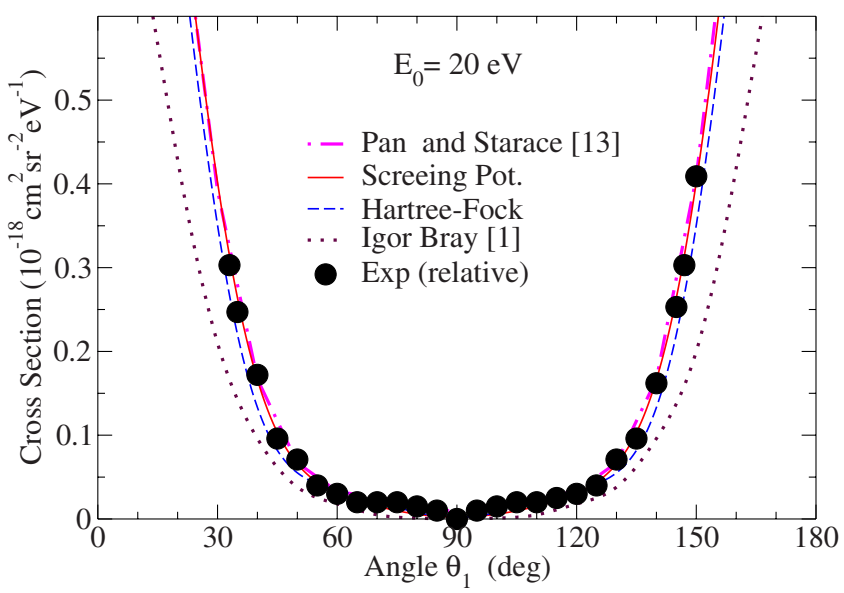

FIG. 4. (Color online) Same as Fig. 1, but for $E_{0}=20 \mathrm{eV}$. 


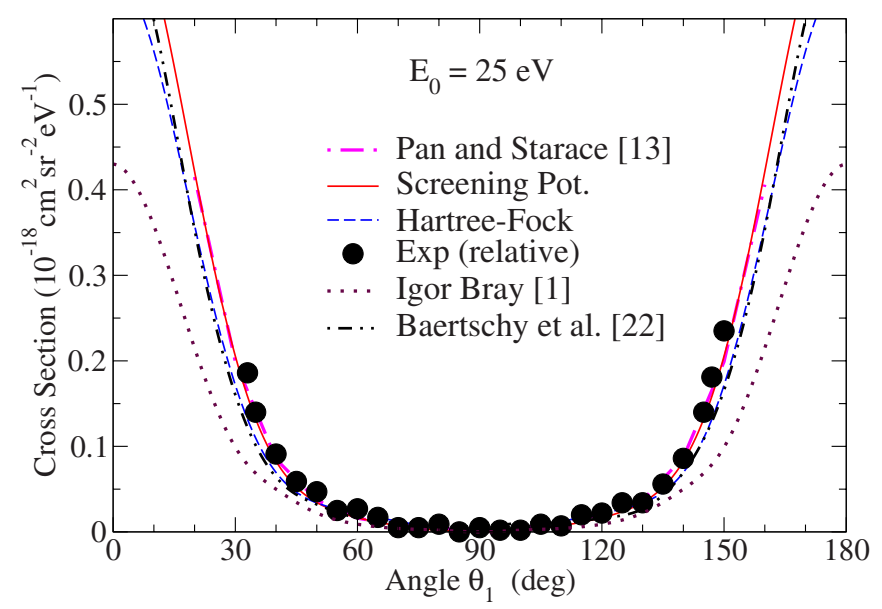

FIG. 5. (Color online) Same as Fig. 1, but for $E_{0}=25 \mathrm{eV}$.

$=90^{\circ}$. For all these energies both our theoretical results describe accurately the measured angular distribution. The cross sections calculated in the screening potential approximation are comparatively in better agreement with experiment than the HF results over all ranges of $\theta_{1}$ measured by experiment. Again at these energies our screening potential results are in very good agreement with those obtained by Pan and Starace [13] using similar approximations, and the CCC results of Bray $[19,20]$. At $25 \mathrm{eV}$ the ECS results of Baertschy et al. [21] agree very well with our present results. Notice that at all these energies considered, there is very little difference between the $\mathrm{HF}$ and the screening potential results and the other available accurate CCC, ECS, and TDCC results where the effect of electron correlation have been taken into account. This implies that in the case of the $\mathrm{H}$ target the effect of distortion between the two outgoing electrons is small even at low energies in the present configuration when the two electrons are emitted in the opposite direction. Here it has been shown that the HF approximation at these energies has been able to describe measured triple differential cross sections at energies very close to threshold.

As stated earlier, we have performed calculation in the two approximations, i.e., the HF approximation and the screening potential approximation, to determine the effect of distortion of the two final state continuum electron wave functions. The HF approximation is applied to the electronimpact ionization of the $\mathrm{H}$ atom at very low energies close to threshold for the geometry considered here. The screening potential approximation used in the present calculation is similar to the one used by Pan and Starace, the only difference being the numerical methods used in the two calculations. The purpose of using the screening potential approximation is to check our computer code and to reproduce their results and also to show that the results obtained by the screening potential approximation, where the interaction between the two continuum electrons has been taken into account, agrees very well with the HF results where this interaction is ignored. Also the HF results are in very good agreement with other calculated results obtained by sophisticated methods where the electron correlation have been considered. Moreover, it has been shown that the HF approximation has been able to reproduce the measured triple differential cross section at energies very close to threshold demonstrating that when the two final state continuum electrons are ejected in the opposite direction, the effect of distortion between them is very small.

\section{CONCLUSION}

We have investigated electron-impact ionization of the $\mathrm{H}$ atom using the recently extended MCHF method [15] to calculate triple differential cross sections for the final state electrons sharing equal energy and for coplanar $\theta_{12}=\pi$ geometry. In this paper we presented results of our calculation for several incident electron energies $E_{0}=14.6,15.6,17.6,20$, and $25 \mathrm{eV}$ in order to compare with absolute and relative experimental $[13,14]$ and other available theoretical results. We used HF and screening potential approximations to determine the importance of distortion between the two final state continuum electrons. We found that at incident energies $E_{0}$ $=15.6$ and $17.6 \mathrm{eV}$ both our theoretical results are very close to each other and are in very good agreement with absolute measurements [13] and the available accurate results of Bray $[19,20]$, Baertschy et al. [21], and Colgan and Pindzola [22]. This indicates that the distortion effect between the two outgoing electrons is very small for the configuration considered here even at these low energies. At all other energies both our HF and screening potential results agree very well with relative experimental observation [13] and other available theoretical results $[13,20,21]$. Both of our theoretical results have minima at $\theta_{1}=\frac{\pi}{2}$ in excellent agreement with experiment. At $E_{0}=14.6$ and $25 \mathrm{eV}$ both our results agree very well with the relative experimental measurements and also with each other for ranges of $\theta_{1}$ considered by experiment. We found that our results calculated in the screening potential approximation agree very well with the distorted partial wave results of Pan and Starace who used similar approximation. Our investigation shows that the present HF results where electron correlations are absent are in good agreement with experimental measurements and other available accurate theoretical results where distortion effects directly or indirectly have been taken into account. This confirms that in the present configuration where two final state continuum electrons leave in the opposite direction, the effect of electron correlation between the two continuum electrons is very small at these low energies considered.

Since the MCHF method is capable of solving coupled integrodifferential equations for excited bound and many continuum channels self-consistently completely numerically in the MCHF approximation, one can systematically take into account the electron correlation and polarization effects completely $a b$ initio through the configuration interaction procedure. Moreover, since at low energies the electron correlation and polarization effects are very important particularly close to threshold this method should be able to compute the cross section very accurately near the threshold.

Because of our success in the extension of the multichannel MCHF method [23] to allow calculation of the triple 
differential cross section for electron-impact ionization of atoms and ions we believe that the extended MCHF method [15] will be successfully able to treat electron correlation and polarization effects which are extremely important particularly at low energies. In the near future we intend to extend our investigation on the triple differential cross section for electron-impact ionization processes in He. Later we would like to include the electron correlation and polarization

[1] I. Bray, J. Phys. B 33, 581 (2000).

[2] T. N. Rescigno, M. Baertschy, W. A. Isaacs, and C. W. McCurdy, Science 286, 2474 (1999).

[3] J. Colgan, M. S. Pindzola, F. J. Robicheaux, D. C. Griffin, and M. Baertschy, Phys. Rev. A 65, 042721 (2002).

[4] K. Bartschat and P. G. Burke, J. Phys. B 20, 3191 (1987).

[5] D. H. Madison, R. V. Calhoun, and W. N. Shelton, Phys. Rev. A 16, 552 (1977).

[6] Cheng Pan and A. F. Starace, Phys. Rev. Lett. 67, 185 (1991); Phys. Rev. A 45, 4588 (1992).

[7] M. Brauner, J. S. Briggs, and H. Klar, J. Phys. B 22, 2265 (1989).

[8] M. Brauner, J. S. Briggs, H. Klar, J. T. Broad, T. Rosel, K. Jung, and H. Ehrhardt, J. Phys. B 24, 657 (1991).

[9] A. Temkin, Phys. Rev. 126, 130 (1962).

[10] R. Poet, J. Phys. B 11, 3081 (1978).

[11] A. Temkin, Phys. Rev. Lett. 49, 365 (1982).

[12] P. Schlemmer, T. Rosel, K. Jung, and H. Ehrhardt, Phys. Rev. Lett. 63, 252 (1989).

[13] J. Roder, H. Ehrhardt, Cheng Pan, Anthony F. Starace, Igor Bray, and Dmitry V. Fursa, Phys. Rev. Lett. 79, 1666 (1997). effects into the initial and final state wave functions to study their effects into the triple differential cross sections.

\section{ACKNOWLEDGMENT}

We are grateful to Dr. A. F. Starace for many valuable discussions and constant help in this connection.
[14] J. Roder, M. Baertschy, and Igor Bray, Phys. Rev. A 67, 010702(R) (2003).

[15] H. P. Saha, J. Phys. B 41, 055201 (2008).

[16] M.R.H. Rudge and M. J. Seaton, Proc. R. Soc. London, Ser. A 283, 262 (1965).

[17] R. K. Peterkop, Theory of Ionization of Atoms by Electron Impact (Colorado Associated University Press, Boulder, CO, 1977), pp. 128 and 129

[18] S. Jetzke, J. Zaremba, and F. H. M. Faisal, Z. Phys. D 11, 63 (1989); F. H. M. Faisal, in Atoms in Strong Fields, edited by C. A. Nocolaides, C. W. Ckark, and M. H. Nayfeh (Plenum, New York, 1990), pp. 407-424.

[19] Igor Bray, J. Phys. B 32, L119 (1999).

[20] I. Bray, Phys. Rev. Lett. 89, 273201 (2002).

[21] M. Baertschy, T. N. Rescigno, and C. W. McCurdy, Phys. Rev. A 64, 022709 (2001).

[22] J. Colgan and M. S. Pindzola, Phys. Rev. A 74, 012713 (2006).

[23] H. P. Saha and D. J. Murray, J. Phys. B 38, 3015 (2005).

[24] H. P. Saha, Phys. Rev. Lett. 65, 2003 (1990).

[25] C. Froese Fischer, Comput. Phys. Commun. 14, 145 (1978). 